\title{
Nutrition risk among an ethnically diverse sample of community-dwelling older adults
}

\author{
Patricia Sheean ${ }^{1, *}$, Isabel C Farrar ${ }^{2}$, Suela Sulo ${ }^{3}$, Jamie Partridge ${ }^{3}$, Linda Schiffer ${ }^{4}$ and \\ Marian Fitzgibbon ${ }^{4,5}$ \\ 'Marcella Niehoff School of Nursing, Loyola University Chicago, 2160 South First Avenue, Building 120, Room \\ 4527, Maywood, IL 60153, USA: ${ }^{2}$ Survey Research Laboratory, University of Illinois at Chicago, Chicago, IL, USA: \\ ${ }^{3}$ Abbott Nutrition Research \& Development, Columbus, OH, USA: ${ }^{4}$ Institute for Health Research and Policy, \\ University of Illinois at Chicago, Chicago, IL, USA: ${ }^{5}$ Department of Pediatrics, College of Medicine, University of \\ Illinois at Chicago, Chicago, IL, USA
}

Submitted 13 March 2018: Final revision received 14 September 2018: Accepted 25 September 2018: First published online 8 November 2018

\begin{abstract}
Objective: To assess the prevalence of nutritional risk among an ethnically diverse group of urban community-dwelling older adults and to explore if risk varied by race/ethnicity.

Design: Demographic characteristics, Katz's activities of daily living and healthcare resource utilization were ascertained cross-sectionally via telephone surveys with trained interviewers. Nutrition risk and nutrition symptomology were assessed via the abridged Patient Generated Subjective Global Assessment (abPG-SGA); scores of $\geq 6$ points delineated 'high' nutrition risk. Descriptive statistics and logistic regression analyses were conducted.

Setting: Urban.

Participants: White, Black or Hispanic community-dwelling adults, $\geq 55$ years of age, fluent in English or Spanish, residing in the city limits of Chicago, IL, USA.

Results: A total of 1001 participants (37\% white, 37\% Black, 26\% Hispanic) were surveyed. On average, participants were 66.9 years old, predominantly female and overweight/obese. Twenty-six per cent $(n$ 263) of participants were classified as 'high' nutrition risk with 24, 14 and $31 \%$ endorsing decreased oral intake, weight loss and compromised functioning, respectively. Black respondents constituted the greatest proportion of those with high risk scores, yet Hispanic participants displayed the most concerning nutrition risk profiles. Younger age, female sex, Black or Hispanic race/ethnicity, emergency room visits, eating alone and taking three or more different prescribed or over-the-counter drugs daily were significantly associated with high risk scores $(P<0.05)$.

Conclusions: One in four older adults living in an urban community prone to health disparities was classified as 'high' nutrition risk. Targeted interventions to promote healthy ageing are needed, especially for overweight/obese and minority community members.
\end{abstract}

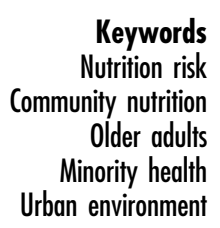

Compromises in nutritional status can be a challenge to healthy ageing among community-dwelling older adults ${ }^{(1)}$. In contrast to the numerous reports from acute care settings, the prevalence of nutritional risk in the community setting is less well established. This is somewhat surprising, considering the growth of the ageing population, the resultant mounting chronic disease burden and the simple fact that most individuals live independently (i.e. not in an acute or long-term care setting). Nevertheless, from a practical standpoint, assessing nutrition risk in the community setting is complex. First, there are a multitude of nutrition screening tools available. Therefore selecting the most appropriate is not necessarily straightforward, as each captures different risk profiles of target populations and has varying reliability ${ }^{(2,3)}$. Second, from an economic standpoint, it is a challenge to provide dedicated staff time to administer a nutrition screening tool, especially in a busy primary care office or geriatrics practice. Finally, in the event the nutrition screen identifies problems that could require immediate intervention or follow-up, access to appropriate resources in the community setting may be a challenge for patients and/or clinical staff (e.g. registered 
dietitians, affordable foods, supplements, clinical testing, etc.). Regardless, nutrition screening is considered a simple, yet critical first step in identifying if there are sufficient risk factors to warrant a more in-depth nutrition assessment, which includes physical examination and functional measures to substantiate a diagnosis of malnutrition ${ }^{(4)}$.

Several investigators have examined nutrition risk within the community setting using different approaches; however, most of the investigations have focused on free-living adults outside the USA. For example, Kvamme et al. ${ }^{(5)}$ evaluated the associations between $\mathrm{Zn}$ status and the risk of malnutrition in a cross-sectional sample of 1521 persons from Norway between the ages of 65 and 87 years. Using the Malnutrition Universal Screening Tool (MUST) and requiring participants to travel to the study centre for evaluation, $8 \%$ ( $n$ 122) of participants were classified 'at nutritional risk'. Of these, $10 \%(n 81)$ were women and $5.5 \%(n$ 41) were men. Westergren et al. ${ }^{(6)}$ evaluated the frequency of malnutrition risk and falling in 465 Swedish persons (age range 73-90 years). Data were collected during preventive home visits and interviews were conducted by two trained visiting research nurses. These authors reported that $35 \%$ of these individuals were at moderate nutritional risk and $30 \%$ were at high nutritional risk after administering the Seniors in the Community: Risk Evaluation for Eating and Nutrition Questionnaire (SCREEN II). Further, Winter et al. ${ }^{(7)}$ reported that after the administration of the Mini Nutrition Assessment - Short Form (MNA-SF) in a general health clinic for community participants attending the ' 75 plus' health clinic in Australia, 16\% ( $n$ 36) were deemed 'at nutritional risk'. Although mean BMI was lower in those at risk, $34 \%$ of those in the at-risk group were overweight or obese (BMI $\geq 25 \cdot 0$ $\mathrm{kg} / \mathrm{m}^{2}$ ). These studies highlight the variability among nutrition screening tools, the resources needed to gather these data for individuals living in the home setting and the lack of diversity of populations sampled. These factors pose limitations to applying and/or generalizing these findings to more diverse populations of community-dwelling older adults, especially within the USA.

Therefore, the purpose of the present telephone-based study was to assess the nutrition risks of an ethnically diverse group of community-dwelling older adults and to explore if risk varied by race/ethnicity. Unlike previous studies, we sought to obtain these data using resourceefficient methodologies that did not warrant in-person administration, physical examination and/or participant travel to a central location. We hypothesized that nutrition risk would be greatest among Black respondents compared with respondents of other race/ethnicities due to greater overall chronic disease burden ${ }^{(8)}$.

\section{Methods}

The current cross-sectional study was conducted in collaboration with the Survey Research Laboratory (SRL) at the University of Illinois at Chicago. Data were collected by the Interviewing Service of America (ISA). The study was conducted specifically in Chicago, IL, USA; a homogeneous urban environment rich in ethnic variability and health disparities ${ }^{(9)}$.

\section{Interviewer training}

Prior to participant enrolment, twenty-nine ISA interviewers and supervisors were trained by the SRL project coordinator. The training included a general orientation to the design and purpose of the study, instructions on gaining cooperation of the respondent, a question-byquestion review of the survey instrument (with instructions on how to record answers and how to probe) and practice interviews. All field staff were supplied with an interviewer training manual covering all aspects of the data collection procedures. In addition, all interviewers attended an in-depth debriefing before and during data collection, which included additional review of the study objectives/background, practice interviews and constructive feedback.

\section{Field procedures}

Interviews were conducted over several months by English- and Spanish-speaking, trained ISA interviewers. Four sample frames were included in the study sample: two listed sample frames (cell and landline) and two random-digit-dial sample frames (cell and landline). The random-digit-dial sample included unlisted numbers. The two listed phone frames were targeted listed samples; targeted to attain completed interviews with people in the needed age range and racial/ethnic groups. The two random-digit-dial frames included a randomly selected sample of cellular and landline phone numbers in the City of Chicago. Cellular and landline random-digit-dial phone numbers were included to give everyone (or, all those who have any type of phone associated with the City of Chicago) some probability of being included in the sample. This methodology increases population coverage over a design that includes only the listed sample or only the random-digit-dial sample. Calls to potential participants were prioritized to weekday evenings and weekends to increase the probability of successful contact with respondents. Up to six call attempts were made before finalizing a case as a 'non-contact'.

\section{Participant eligibility and inclusion}

Initial eligibility was established once the participant affirmed the following criteria: (i) 55 years of age or greater; (ii) self-reported White, Black or Hispanic; (iii) able to speak and understand English or Spanish; and (iv) currently living in the city limits of Chicago, IL, USA. Participants were included if they were willing to complete the remainder of the telephone survey or if they possessed 
a cellular phone with a Chicago area code but resided outside the geographic city limits.

\section{Questionnaire administration}

Respondents were informed that the interview would require approximately $20 \mathrm{~min}$ to complete and incentives of \$US 10 would be provided, unless the respondent declined to provide his/her mailing address. In addition to demographic items, respondents were asked about whom they resided with as well as access to and type of healthcare insurance.

\section{Nutritional risk assessment}

To assess nutritional risk, interviewers administered the abridged Patient Generated Subjective Global Assessment (abPG-SGA), a screening tool typically used in the oncology setting ${ }^{(10)}$. However, unlike the $\mathrm{MNA}^{(11)}$, the abPG-SGA tool foregoes the physical exam as well as the disease condition and metabolic considerations of the conventional PG-SGA ${ }^{(12)}$. The abPG-SGA is unique in that it affords the opportunity to capture specific nutrition impact symptoms (e.g. anorexia, constipation, dry mouth, etc.). These symptoms are not captured in screening tools conventionally employed in community populations, such as the MUST $^{(5)}$, DETERMINE ${ }^{(13)}$ or Short Nutritional Assessment Questionnaire 65+ $(\mathrm{SNAQ} 65+)^{(14)}$, yet they are clinical important, relevant and easily administered over the phone. Specific symptoms, including weight loss, changes in oral intake and global decreases in physical functioning, as well as other responses are assigned numeric values, where higher scores reflect greater nutrition risk. Participants can complete the abPG-SGA in a minimum of eight or a maximum of twenty-three queries, depending upon current symptomology. Scores of $\geq 6$ on the abPG-SGA are highly correlated with patients classified as malnourished when the full versions of the PG-SGA (97\% sensitivity, 86\% specificity, area under the curve $=$ 0.967) and the Malnutrition Screening Tool (81\% sensitivity, $72 \%$ specificity, area under the curve $=0.823$ ) are employed $^{(10)}$. In addition, we asked if participants 'ate alone most of the time' as a proxy of social isolation.

\section{Functional assessment}

Respondents were asked about potential functional impairments. Using the framing of Katz, participants were asked to rate themselves as dependent (i.e. needing supervision, direction, personal assistance or total care) or independent (i.e. no supervision, direction or personal assistance) in performing six activities of daily living ${ }^{(15)}$. Scores range from 0 to 6 ; higher scores indicate participant independence.

\section{Health-care utilization}

To explore potential associations between nutrition risk factors and health-care resources, respondents were asked a sequence of questions pertaining to health-care services received in the last 6 months. Participants were asked to respond to inquiries regarding: visits to the emergency room (frequency and medical reason), hospital, skilled nursing and/or inpatient rehabilitation facility admissions (frequency and admitting diagnosis), and the receipt of home care services (yes/no and if yes, rationale for services). In an attempt to get at the concept of polypharmacy, we also asked if participants 'took three or more different prescribed or over-the-counter drugs per day'. This wording is supported by the DETERMINE tool ${ }^{(13)}$.

\section{Data processing and statistical analyses}

Open-ended text data were translated from Spanish to English and all data were back-coded, which involved reviewing the all open-ended responses to determine if any could be coded into pre-existing response categories. Sample weights were applied to match the American Community Survey data based on 5-year estimates from 2011-2015 for age (55-64 and 65-75 years), gender (male or female) and by overall distribution of race/ethnicity (White, Black and Hispanic) in the City of Chicago ${ }^{(16)}$. Descriptive statistics were completed to: (i) examine participant characteristics and nutrition risk scores stratified by race/ethnicity; and (ii) assess the prevalence of nutrition risk symptoms stratified by race/ethnicity Additionally, we conducted some exploratory analyses to see if nutrition risk differed by sex and if obesity (defined as BMI $\geq 30.0 \mathrm{~kg} / \mathrm{m}^{2}$ ) was associated with specific nutrition risk factors among those deemed at high risk. Two-sample $t$ tests were used for continuous variables and Pearson and $\chi^{2}$ tests were conducted for categorical variables. Logistic regression analyses were conducted to determine the characteristics that independently predicted nutrition risk, taking account of significant covariates in the bivariate analyses and multicollinearity. All statistical analyses were conducted using the statistical software package SAS version 9.4 and a $P$ value of 0.05 was used to denote statistical significance.

\section{Results}

Of the 64445 relevant phone numbers within the ISA database, the majority were classified as 'no longer working' ( $42 \%, n 27022$ ), 'no answer' (27\%, n 17 427), 'answering machine/voicemail' (19\%, $n$ 11922) or 'now living outside the City of Chicago' ( $2 \%, n$ 1010). Of the 7064 remaining numbers, $20 \%$ ( $n$ 1430) were 'ineligible', $48 \%$ ( $n$ 3402) were 'not available/unable to screen', $15 \%$ ( $n$ 1081) 'refused either before or after screening' and $2 \%$ ( $n$ 150) were screened but unable to interview. Two sets of response rates were calculated for the study. Using a ratio of completed interviews ( $n$ 1001) to the sum of cases known to be eligible ( $n$ 1166) and estimates of the number 
of eligible cases among the unknown eligible ( $n$ 11783), the conservative resulting response rate was $7 \cdot 7 \%$. However, if we included only the individuals who answered and cooperated with the screening process, our response rate was $85.8 \%$ ( $n$ 1001/1166). The average number of call attempts per participant was $2 \cdot 1$. Interviews averaged $12.3 \mathrm{~min}$ in length and were conducted by ISA, in English or Spanish. Of the 1001 respondents who completed the interview, $845(84.4 \%)$ agreed to provide their mailing address to receive the incentive.

In general, the average study participant was 66.9 (SD 6.4) years of age, predominantly female (69\%), classified as overweight using BMI $\left(29.5(\mathrm{sD} 6 \cdot 7) \mathrm{kg} / \mathrm{m}^{2}\right)$, educated (24\%, n 240 completed some college; 35\%, n 355 possessed a college and/or graduate degree), and largely independent with regard to activities of daily living (91\% reported autonomy in all six activities). For all participants, $17 \%$ ( $n$ 172) reported visits to the emergency room, $13 \%$ ( $n$ 128) were admitted to a hospital and 10\% ( $n$ 104) required home care services within the last 6 months. Further, $48 \%$ ( $n$ 481) reported eating alone most of the time and nearly $56 \%$ ( $n$ 559) reported taking three or more different prescribed or over-the-counter drugs daily.

Table 1 displays the characteristics of the study participants stratified by nutrition risk scores, revealing that $26 \%$ ( $n$ 263) of participants were classified as high risk $(\geq 6$ points). These high-risk individuals were more likely to be female $(P=0 \cdot 01)$, minority $(P<0 \cdot 0001)$, less educated $(P<0.0001)$ and obese $(P=0 \cdot 01)$. Additionally, participants classified as high risk were less independent in their activities of daily living (all $P<0 \cdot 05$ ), reported significantly higher health-care utilization, more frequently ate alone $(P<0.0001)$ and more frequently took three or more different prescribed or over-the-counter drugs daily $(P<0 \cdot 0001)$. Specifically, the prevalence of nutrition risk for White, Black and Hispanic participants was 16\% ( $n$ 60/ 373), 34\% ( $n$ 124/369) and $31 \%$ ( $n$ 79/259), respectively. In a separate subgroup analyses of participants deemed at high nutrition risk, we examined if BMI was associated with any of the nutrition risk variables listed in Table 1 . Although more females were classified as obese ( $n$ 93/ $251, P=0 \cdot 02)$, BMI was not associated with nutrition risk. BMI was significantly associated with taking three or more different prescribed or over-the-counter drugs daily $(P=0.002)$.

Table 2 presents the results of the logistic regression analyses conducted to predict nutrition risk. Younger age, female sex, minority race/ethnicity, emergency room visits, eating alone and taking three or more different prescribed or over-the-counter drugs daily were significantly associated with high nutrition risk, when adjusted for all other predictors included in the model. We tested the covariates for multicollinearity in the logistic regression model by examining the covariance matrix as well as the variance inflation factor and tolerance diagnostics. All correlation coefficients were $<0 \cdot 5$, the variance inflation factor was $<1.7$ for all covariates and the tolerance was $>0.6$ for all covariates; thus, multicollinearity was not a significant concern for this model. Education did not appear to be significantly associated with nutrition risk overall $(P=0.09)$, but participants with some college appeared to be at higher risk than college graduates $(P=0.02)$.

Table 3 examines specific components of the abPG-SGA by race/ethnicity and sex. Compared with White participants, Black participants reported eating less than usual $(P<0.001)$ and experienced significantly more nutrition symptomology related to decreased appetite $(P<0 \cdot 001)$, constipation $(P<0 \cdot 001)$, taste changes $(P<0 \cdot 001)$, bothersome smells $(P<0.001)$, early satiety $(P<0.05)$ and pain $(P<0.05)$. Compared with White respondents, Hispanic respondents reported significantly more nutrition symptomology related to decreased appetite $(P<0.05)$, constipation $(P<0.001)$, dry mouth $(P<0 \cdot 01)$, taste changes $(P<0.001)$, bothersome smells $(P<0.001)$, early satiety $(P<0.01)$ and fatigue $(P<0 \cdot 01)$. Of the fifty-nine Hispanic participants who reported 'less than usual intake', they were consuming significantly little solid food $(P<0 \cdot 001)$, liquids $(P<0.05)$, only nutrition supplements $(P<0.01)$ and very little of anything $(P<0 \cdot 001)$, compared with the sixty-three White participants who reported 'less than usual intake'. Black and Hispanic respondents reported significantly decreased physical functioning ('not feeling up to most things', $P<0.05$; 'able to do little activity/bedridden', $P<0.001)$ compared with White respondents. When these nutrition variables were stratified by sex (male $v$. female), females reported a higher frequency of 'no appetite' $(P=0.02)$ and 'smells bothersome' $(P=0.003)$ compared with males.

\section{Discussion}

In the present study which relied upon self-reported data, we found that $26 \%$ of individuals who responded to our telephone survey had nutrition risk scores that would likely correlate with malnutrition classification ${ }^{(10)}$. Nutrition risk was higher among minority compared with nonminority participants, as evidenced by the significantly higher frequency of nutrition symptomology and decreased global physical functioning. Consistent with our hypothesis, Black respondents displayed a higher nutrition risk profiles compared with persons of other race/ethnicity, making an important contribution to the literature since previous studies have included predominantly nonminority participants. Similar to other studies, we also found that females were more likely to be at nutrition risk overall compared with men ${ }^{(17,18)}$. Because our sampling strategies only accounted for race/ethnicity and not sex, we cannot say if this reflects a true difference in nutrition risk or if females were more likely to respond to our survey requests. When we analysed the individual nutrition 
Table 1 Characteristics of urban community-dwelling older adults at low and high nutritional risk†, Chicago, IL, USA, August-October 2017

\begin{tabular}{|c|c|c|c|c|c|c|c|}
\hline \multirow[b]{2}{*}{ Variable } & \multicolumn{3}{|c|}{$\begin{array}{l}\text { Low nutrition risk (<6 points) } \\
\qquad(N 738)\end{array}$} & \multicolumn{3}{|c|}{$\begin{array}{l}\text { High nutrition risk ( } \geq 6 \text { points) } \\
\qquad(N 263)\end{array}$} & \multirow[b]{2}{*}{$P \ddagger$} \\
\hline & $N$ & Mean or $n$ & SD or $\%$ & $N$ & Mean or $n$ & SD or $\%$ & \\
\hline Age (years) & 697 & $67 \cdot 2$ & $6 \cdot 4$ & 255 & $66 \cdot 3$ & $6 \cdot 3$ & 0.06 \\
\hline Sex & 738 & & & 263 & & & 0.01 \\
\hline Male & & 242 & 33 & & 65 & 25 & \\
\hline Female & & 496 & 67 & & 198 & 75 & \\
\hline Race/ethnicity & 738 & & & 263 & & & $<0.0001$ \\
\hline White & & 313 & 42 & & 60 & 23 & \\
\hline Black & & 245 & 33 & & 124 & 47 & \\
\hline Hispanic & & 180 & 24 & & 79 & 30 & \\
\hline Education & 676 & & & 234 & & & $<0.0001$ \\
\hline Not HS graduate & & 100 & 15 & & 51 & 22 & \\
\hline HS graduate or GED & & 121 & 18 & & 43 & 18 & \\
\hline Some college & & 162 & 24 & & 78 & 33 & \\
\hline College graduate & & 293 & 43 & & 62 & 27 & \\
\hline BMI $\left(\mathrm{kg} / \mathrm{m}^{2}\right)$ & 687 & $29 \cdot 1$ & $6 \cdot 3$ & 251 & $30 \cdot 6$ & $7 \cdot 6$ & 0.001 \\
\hline BMI category & 687 & & & 251 & & & 0.01 \\
\hline Underweight $\left(<18.5 \mathrm{~kg} / \mathrm{m}^{2}\right)$ & & 3 & $<1$ & & 4 & 2 & \\
\hline Normal weight $\left(18.5-24.9 \mathrm{~kg} / \mathrm{m}^{2}\right)$ & & 192 & 28 & & 52 & 21 & \\
\hline Overweight $\left(25.0-29.9 \mathrm{~kg} / \mathrm{m}^{2}\right)$ & & 247 & 36 & & 81 & 32 & \\
\hline Obese $\left(\geq 30.0 \mathrm{~kg} / \mathrm{m}^{2}\right)$ & & 245 & 36 & & 114 & 45 & \\
\hline \multicolumn{8}{|l|}{ Independence with ADL } \\
\hline Bathing & 736 & 718 & 98 & 262 & 246 & 94 & 0.005 \\
\hline Dressing & 737 & 723 & 98 & 263 & 250 & 95 & 0.009 \\
\hline Toileting & 737 & 727 & 99 & 263 & 256 & 97 & 0.17 \\
\hline Transferring & 737 & 721 & 98 & 263 & 244 & 93 & 0.0001 \\
\hline Continence & 730 & 707 & 97 & 260 & 235 & 90 & $<0.0001$ \\
\hline Feeding & 736 & 730 & 99 & 263 & 256 & 97 & 0.049 \\
\hline All 6 ADL & 728 & 683 & 94 & 259 & 219 & 85 & $<0.0001$ \\
\hline \multicolumn{8}{|l|}{ Health-care utilization } \\
\hline ER visit & 736 & 96 & 13 & 262 & 76 & 29 & $<0.0001$ \\
\hline Hospital admission & 735 & 81 & 11 & 263 & 47 & 18 & 0.004 \\
\hline SNF admission & 735 & 11 & 2 & 263 & 6 & 2 & 0.41 \\
\hline Home care services & 737 & 61 & 8 & 262 & 43 & 16 & 0.0002 \\
\hline Eat alone most of the time & 736 & 317 & 43 & 261 & 164 & 63 & $<0.0001$ \\
\hline Three or more different prescribed or OTC drugs per day & 735 & 372 & 51 & 262 & 187 & 71 & $<0.0001$ \\
\hline
\end{tabular}

HS, high school; GED, General Equivalency Diploma; ADL, activities of daily living; ER, emergency room; SNF, skilled nursing facility; OTC, over-the-counter. †Nutritional risk is defined using abridged Patient Generated Subjective Global Assessment (abPG-SGA) score: $<6$ points $=$ low risk; $\geq 6$ points $=$ high risk.

$\ddagger$ Tests for differences between low- and high-risk groups: $t$ tests, with pooled variance for age and BMI, test for row mean score difference for ordinal variables (education and BMI categories); Fisher's exact test for feeding, toileting and SNF admission; and $x^{2}$ tests for other categorical variables. Responses of 'don't know' or 'refused' are treated as missing and excluded from the denominator.

Table 2 Logistic regression analyses for predicting nutritional risk among urban community-dwelling older adults†, Chicago, IL, USA, August-October 2017

\begin{tabular}{|c|c|c|c|c|}
\hline & OR & $95 \% \mathrm{Cl}$ & $b$ & $P$ \\
\hline Intercept & & - & -1.507 & 0.16 \\
\hline Age, year & 0.97 & $0.94,1.00$ & -0.034 & 0.02 \\
\hline Female $($ ref. $=$ male $)$ & 1.69 & $1 \cdot 13,2 \cdot 51$ & 0.522 & 0.01 \\
\hline Race (ref. $=$ White) & & & & 0.0004 \\
\hline Black & 2.41 & $1.55,3.73$ & 0.878 & $<0.0001$ \\
\hline Hispanic & 1.85 & $1.09,3.14$ & 0.616 & 0.02 \\
\hline Education (ref. $=$ college graduate) & & & & 0.09 \\
\hline Not HS graduate & 1.68 & $0.94,3.01$ & 0.521 & 0.08 \\
\hline HS graduate/GED & $1 \cdot 17$ & $0.69,1.98$ & 0.155 & 0.57 \\
\hline Some college & 1.69 & $1.07,2.66$ & 0.523 & 0.02 \\
\hline $\mathrm{BMI}, \mathrm{kg} / \mathrm{m}^{2}$ & 1.01 & $0.99,1.04$ & 0.011 & 0.41 \\
\hline Dependent for $\geq 1 \mathrm{ADL}$ (ref. = independent for all $6 \mathrm{ADL}$ ) & 1.36 & $0.72,2.59$ & 0.308 & 0.35 \\
\hline ER visit (ref. = none) & 2.50 & $1.56,4.00$ & 0.915 & 0.0001 \\
\hline Hospital admission (ref. = none) & $1 \cdot 10$ & $0.63,1.90$ & 0.091 & 0.74 \\
\hline Home care services $($ ref. $=$ none $)$ & 1.22 & $0.69,2 \cdot 14$ & 0.196 & 0.50 \\
\hline Eat alone most of the time (ref. $=$ no) & $2 \cdot 36$ & $1.66,3.36$ & 0.858 & $<0.0001$ \\
\hline Take three or more different prescribed or OTC drugs per day (ref. $=$ no) & 2.33 & $1.59,3.42$ & 0.845 & $<0.0001$ \\
\hline
\end{tabular}

ref., reference category; HS, high school; GED, General Equivalency Diploma; ADL, activities of daily living; ER, emergency room; OTC, over-the-counter. †From a logistic regression model predicting the risk of an abridged Patient Generated Subjective Global Assessment (abPG-SGA) score of $\geq 6$ points (high nutrition risk). Some observations were excluded from the model due to missing data for covariates: $N 810$ (210 were high risk). 
Table 3 Prevalence of nutritional risk symptoms stratified by race/ethnicity and sex, using the components of the abridged Patient Generated Subjective Global Assessment (abPG-SGA), in urban community-dwelling older adults, Chicago, IL, USA, August-October 2017

\begin{tabular}{|c|c|c|c|c|c|c|c|c|c|c|c|c|c|}
\hline \multirow[b]{2}{*}{ Variable } & \multirow[b]{2}{*}{$N$} & \multicolumn{2}{|c|}{$\begin{array}{l}\text { White } \\
(N 373)\end{array}$} & \multicolumn{2}{|c|}{$\begin{array}{c}\text { Black } \\
(N \text { 369) }\end{array}$} & \multicolumn{2}{|c|}{$\begin{array}{l}\text { Hispanic } \\
(N 259)\end{array}$} & \multirow[b]{2}{*}{$P \dagger$} & \multicolumn{2}{|c|}{$\begin{array}{c}\text { Male } \\
(N 307)\end{array}$} & \multicolumn{2}{|c|}{$\begin{array}{l}\text { Female } \\
(N \text { 694) }\end{array}$} & \multirow[b]{2}{*}{$P \ddagger$} \\
\hline & & $n$ & $\%$ & $n$ & $\%$ & $n$ & $\%$ & & $n$ & $\%$ & $n$ & $\%$ & \\
\hline $\begin{array}{l}\text { During the past two weeks } \\
\text { my weight has: }\end{array}$ & 962 & & & & & & & 0.53 & & & & & $0 \cdot 26$ \\
\hline Decreased & & 44 & 12 & 52 & 15 & 36 & 15 & & 44 & 15 & 88 & 13 & \\
\hline Not changed & & 293 & 81 & 270 & 76 & 189 & 77 & & 226 & 75 & 526 & 79 & \\
\hline Increased & & 24 & 7 & 32 & 9 & 22 & 9 & & 30 & 10 & 48 & 7 & \\
\hline Food intake§ & 995 & & & & & & & $<0.0001$ & & & & & $0 \cdot 10$ \\
\hline Less than usual & & 63 & 17 & $113^{\star \star \star}$ & 31 & 59 & 23 & & 62 & 20 & 173 & 25 & \\
\hline Unchanged & & 272 & 73 & 220 & 60 & 177 & 69 & & 211 & 69 & 458 & 66 & \\
\hline More than usual & & 37 & 10 & \multirow{2}{*}{\multicolumn{2}{|c|}{$(N 113)$}} & \multirow{2}{*}{\multicolumn{2}{|c|}{$(N 59)$}} & & 33 & 11 & 58 & 8 & \\
\hline $\begin{array}{l}\text { If less than usual intake, } \\
\text { now consuming: }\end{array}$ & 235 & \multicolumn{2}{|c|}{$(N 63)$} & & & & & & \multicolumn{2}{|c|}{$(N 62)$} & \multicolumn{2}{|c|}{$(N 173)$} & \\
\hline Normal food & 233 & 57 & 93 & 104 & 92 & 57 & 97 & 0.53 & 59 & 95 & 159 & 93 & 0.76 \\
\hline Little solid food & 235 & 16 & 25 & $55^{\star *}$ & 49 & $39^{\star * *}$ & 66 & $<0.0001$ & 31 & 50 & 79 & 46 & 0.56 \\
\hline Only liquids & 235 & 2 & 3 & 3 & 3 & $10^{\star}$ & 17 & 0.003 & 6 & 10 & 9 & 5 & 0.23 \\
\hline Only nutrition supplements & 234 & 3 & 5 & 13 & 12 & $13^{\star \star}$ & 22 & 0.02 & 5 & 8 & 24 & 14 & 0.23 \\
\hline Very little of anything & 228 & 11 & 18 & 34 & 31 & $33^{\star \star *}$ & 57 & $<0.0001$ & 22 & 35 & 56 & 34 & 0.80 \\
\hline Tube or vein feedings & 235 & 1 & 2 & 0 & 0 & 0 & 0 & - & 1 & 2 & 0 & 0 & - \\
\hline \multicolumn{14}{|l|}{ Symptoms } \\
\hline No problems eating & 1000 & 361 & 97 & 353 & 96 & 249 & 96 & 0.82 & 298 & 97 & 665 & 96 & 0.23 \\
\hline No appetite & 998 & 57 & 15 & $101^{\star \star \star}$ & 27 & $56^{\star}$ & 22 & 0.0004 & 52 & 17 & 162 & 23 & 0.02 \\
\hline Nausea & 1000 & 16 & 4 & 24 & 7 & 22 & 8 & 0.10 & 17 & 6 & 45 & 6 & 0.56 \\
\hline Vomiting & 1001 & 5 & 1 & 6 & 2 & 10 & 4 & 0.08 & 6 & 2 & 15 & 2 & 0.83 \\
\hline Constipation & 999 & 21 & 6 & $49^{\star \star \star}$ & $1 \overline{3}$ & $61^{* * *}$ & 24 & $<0.0001$ & 31 & 10 & 100 & 14 & 0.06 \\
\hline Diarrhoea & 1000 & 31 & 8 & 32 & 9 & 20 & 8 & 0.91 & 24 & 8 & 59 & 9 & 0.71 \\
\hline Mouth sores & 1001 & 12 & 3 & 7 & 2 & 4 & 2 & 0.33 & 10 & 3 & 13 & 2 & 0.18 \\
\hline Dry mouth & 1000 & 35 & 9 & 36 & 10 & $47^{\star *}$ & 18 & 0.001 & 31 & 10 & 87 & 13 & 0.27 \\
\hline Things taste funny & 997 & 13 & 4 & $50^{\star \star \star}$ & 14 & $46^{\star \star \star}$ & 18 & $<0.0001$ & 36 & 12 & 73 & 11 & 0.58 \\
\hline Smells bothersome & 994 & 18 & 5 & $47^{\star \star \star}$ & 13 & $33^{\star \star \star}$ & 13 & 0.0005 & 17 & 6 & 81 & 12 & 0.003 \\
\hline Problems swallowing & 1001 & 10 & 3 & 12 & 3 & 9 & 3 & 0.83 & 10 & 3 & 21 & 3 & 0.85 \\
\hline Early satiety & 991 & 102 & 28 & $130^{*}$ & 36 & $102^{\star *}$ & 39 & 0.008 & 92 & 30 & 242 & 35 & 0.13 \\
\hline Pain & 998 & 17 & 5 & $34^{*}$ & 9 & 11 & 4 & 0.01 & 16 & 5 & 46 & 7 & 0.38 \\
\hline Fatigue & 999 & 45 & 12 & 62 & 17 & $56^{\star \star}$ & 22 & 0.007 & 48 & 16 & 115 & 17 & 0.70 \\
\hline Other problems & 1000 & 12 & 3 & 14 & 4 & 7 & 3 & 0.75 & 9 & 3 & 24 & 3 & 0.67 \\
\hline Activities and function & 993 & & & $\star \star \star$ & & ** & & 0.0002 & & & & & 0.74 \\
\hline Normal without limitations & & 277 & 75 & 230 & 63 & 168 & 65 & & 210 & 69 & 465 & 68 & \\
\hline Not my normal self & & 78 & 21 & 87 & 24 & 54 & 21 & & 65 & 21 & 154 & 22 & \\
\hline Not feeling up to most things & & 12 & 3 & 25 & 7 & 17 & 7 & & 17 & 6 & 37 & 5 & \\
\hline Able to do little activity/bedridden & & 3 & 1 & 24 & 7 & 18 & 7 & & 13 & 4 & 32 & 5 & \\
\hline
\end{tabular}

${ }^{*} P<0.05,{ }^{* *} P<0.01$ and ${ }^{* * *} P<0.001$ are used to denote differences of Black and Hispanic participants where White participants are the referent population. All other $P$ values reflect difference across all groups. Responses of 'don't know' or 'refused' are treated as missing and excluded from the denominator. $\dagger$ From logistic regression analyses (multinomial for weight change and ordinal for activities and function).

fFrom tests for row mean score differences for activities and function; Fisher's exact test for variables with any expected cell values $<5$ and $x^{2}$ tests for other variables.

$\S$ For logistic regression and $x^{2}$ analyses, 'unchanged' and 'more than usual' were combined and compared with 'less than usual'.

risk components by sex (Table 3), the nutrition risk profiles for females were comparable to male participants, supporting that this may be an artifact of higher study participation among females.

It is difficult to directly compare our prevalence estimates with others since different nutrition assessment tools were utilized and because our participants were not recruited from a hospital or clinic. However, our findings are similar to those for individuals living in a rural setting ${ }^{(11)}$, higher than those reported in older participants living at home ${ }^{(19,20)}$ and consistent with those of older hospitalized patients ${ }^{(21-23)}$. Together, these findings raise concerns that the nutrition issues of older adults residing in an urban, community setting are not well recognized. Older individuals possess a multitude of nutrition risk factors due to the physiological changes of ageing, including but not limited to: sensory losses (e.g. taste, smell, sight); alterations in gastrointestinal function (e.g. dysphagia, xerostomia, achlorhydria, delayed gastric emptying); adverse body composition changes (i.e. loss of lean mass, strength and function); decreased cognition; and the side-effects of medications often used to treat chronic diseases ${ }^{(24)}$. Considering the recent US Census Bureau report that the number of older individuals $(\geq 65$ years of age) residing in the USA grew by $40 \%$ from 2000 to $2016^{(25)}$, our findings have significant public health implications. The Institute of Medicine identifies nutrition and the coordination of nutrition services in the community setting as integral components for promoting healthy ageing among older adults ${ }^{(26)}$. 
The most recent consensus report by the Global Leadership Initiative on Malnutrition recommends the use of phenotypic and aetiological criteria be applied to diagnosis malnutrition ${ }^{(27)}$. The aetiology-based diagnosis classification includes malnutrition related to chronic disease with inflammation, chronic disease with minimal or no perceived inflammation, malnutrition related to acute disease or injury with severe inflammation or malnutrition related to starvation. Although limited by our methodologies, we speculate chronic-disease related malnutrition would be the most prevalent among our participants with high nutrition risk scores. Previous work supports that polypharmacy is associated with greater nutrition risk $^{(28-30)}$. The majority of our participants $(56 \%)$ reported taking three or more different prescribed or over-thecounter drugs daily, lending support to the use of this crude metric as a proxy measure of co-morbid conditions and underlying chronic disease burden. In disease-related malnutrition, inflammation leads to a milieu of cytokine responses ${ }^{(31)}$, which adversely impact metabolism, appetite, dietary intake and body composition. Interestingly, we found that symptoms of 'decreased weight', 'no appetite' and 'food intake less than usual' were highly prevalent among participants (Table 3), which are clinical features that conventionally support a malnutrition diagnosis ${ }^{(27)}$. Additionally, $32 \%$ ( $n$ 318) of participants reported limitations in their global performance status, which was further supported by an overall decrease in independence in activities of daily living among those with high nutrition risk scores. Given the high prevalence of obesity in our sample (36\%) and the inability of BMI to discriminate those who were high risk, these data are particularly worrisome for sarcopenic obesity; an occult condition of low lean mass and compromised function in the setting of obesity $\left(\mathrm{BMI} \geq 30 \cdot 0 \mathrm{~kg} / \mathrm{m}^{2}\right)^{(32)}$. Using nationally representative data and dual-energy X-ray absorptiometry to quantify lean mass (kilograms), $24 \%$ of older ( $\geq 60$ years of age) adults residing in the community setting had sarcopenic obesity ${ }^{(33)}$. While nutrition screening would seem a logical first step to identifying individuals at risk, there tends to be a common perception that malnutrition is a problem restricted to the acute care setting and underweight patient populations ${ }^{(34-36)}$. Therefore, it is essential to raise awareness regarding the importance of identifying nutrition risks in both underweight and overweight adults, and further to reducing barriers to intervening through comprehensive nutrition-focused programmes implemented in community space or outpatient clinics. The Global Leadership Initiative on Malnutrition advocates assessing nutrition risks in common arenas (e.g. hospitals and nursing homes), as well as other health-care points of care $^{(37)}$. Our data suggest the emergency room may be one unconventional venue to initiate screening and nutrition interventions in older individuals at high nutrition risk. Additionally, routine nutrition intervention and follow-up after hospitalization for high-risk individuals (i.e. critical illness, prolonged length of stay, etc.) or in those diagnosed with malnutrition seems paramount.

A recent systematic review by Hamirudin et al. ${ }^{(38)}$ highlights outcomes related to nutrition screening and nutrition interventions in older community-living adults. Over a span of 20 years, eleven nutrition interventions were identified and reviewed ${ }^{(28,29,39-47)}$; the prevalence of malnutrition was approximately 35\%. Nutrition interventions in these studies included access to or the provision of: healthy eating flyers ${ }^{(28,29,42,46)}$, nutrition counselling (in person or telephone $)^{(1,39,41,43-45)}$, nutrition education (dietitian access, cooking demonstration) ${ }^{(47)}$ and/or referral to social and community services (e.g. Meals on Wheels, community meal programme $)^{(27,29,40,41,43,44)}$. Hamirudin et al. concluded that timely nutrition screening followed by intervention improves the nutritional status of these older adults. As a follow-up to this work, a pilot study was conducted by Hamirudin et al. ${ }^{(48)}$ in sixty-eight patients to determine if a model of home-based dietetic care improved dietary intake and weight status in a specific group of older adults ( $>65$ years of age) following hospitalization. Individualized diet advice was provided by a dietitian as the primary intervention. Improved nutritional status was demonstrated by increases in body weight $(P=0.048)$, as well as mean MNA scores (21.9 (SD 3.5) v. $25 \cdot 2$ (SD 3.1); $P<0 \cdot 001)$. Specifically, the use of oral nutrition supplements and milk was associated with increased overall energy intake pre- and post-intervention. Recent data also suggest oral nutrition supplementation in community-dwelling older individuals at nutrition risk may be a simple, cost-effective nutrition intervention ${ }^{(49)}$.

Our data provide a unique opportunity to gain insights into the nutritional concerns of an ethnically diverse group of older adults residing within an urban community. We employed methods that posed minimal participant burden and gathered data on a large number of individuals in a relatively short period of time ( $\sim 4$ months). Despite these methodological strengths, the study is not without limitations. First, individuals who refused to participate in our survey may be inherently different. By design, we know very little about those who refused or the ones who could not be reached by phone. Therefore, we cannot determine the degree to which non-response is biasing the results or the true generalizability of our findings. We chose a very conservative method to calculate overall response rate, basing our estimates on the sample of 64445 potential telephone numbers. Using other less conservative methods (e.g. removing non-working numbers, only including direct contact to screener, cooperation with screener and those meeting all eligibility in the denominator) would reflect significantly higher response rates. Second, we did not gather data on depression or social support, two potentially important confounders of nutrition risk ${ }^{(28-30)}$ that would have added substantial time to the length to the interview. Although we did find that 'eating alone' was a major predictor of nutrition risk and this may be a proxy 
for social isolation, a recent systematic review found these two factors were not associated with documented proteinenergy malnutrition in older adults ${ }^{(50)}$. Third, we used the abPG-SGA tool to assess the nutrition risk. This screening tool is conventionally used in the oncology setting and like other screening tools, does rely on self-reported data which then merits corroboration for further nutrition assessment. Fourth, based on the number of available covariates, the likelihood of residual confounding cannot be ruled out. Although there were 210 events (i.e. highrisk individuals), our logistic regression model was limited to fourteen predictors following the statistical methodologies of Peduzzi et al ${ }^{(51)}$. Finally, our cross-sectional study design prohibits the establishment of cause-and-effect relationships.

\section{Conclusions}

The present study documents that $26 \%$ of older adults with overweight and obesity residing in an urban, community environment report signs and symptoms consistent with high nutrition risk. Additionally, Black respondents reflect the greatest proportion of those with high risk scores, yet Hispanic participants display the most concerning nutrition risk profiles. The study contributes to a growing body of research that supports the elevated nutrition risk among independent-living older adults, regardless of BMI, and offers unique findings among minority participants. It is clear that our current identification and intervention strategies are lacking, since the relative majority of research endeavours related to nutrition risk and malnutrition have focused on the inpatient hospital environment. Future studies will require comprehensive and in-depth assessment methods and, more importantly, targeted well-designed interventions to promote healthy ageing throughout our communities.

\section{Acknowledgements}

Financial support: This study was funded by a grant from Abbott Nutrition (\#HA28). I.C.F., M.F. and P.S. received salary support from Abbott Nutrition. S.S. and J.P. are employees of Abbott Nutrition. P.S. was also supported by the Gannon Center for Women and Leadership, Loyola University Chicago. Conflict of interest: None declared. Authorship: I.C.F., M.F., J.P., P.S. and S.S. contributed to the conception and design of the research; I.C.F. and L.S. contributed to the acquisition and analysis of the data; I.C.F., M.F., L.S. and P.S. contributed to the interpretation of the data; M.F. and P.S. drafted the manuscript. All authors critically revised the manuscript, agreed to be fully accountable for ensuring the integrity and accuracy of the work, and read and approved the final manuscript. Ethics of buman subject participation: This study was conducted according to the guidelines laid down in the Declaration of Helsinki and all procedures involving human subjects were approved by the University of Illinois at Chicago and Loyola University Chicago Institutional Review Boards. Verbal informed consent was obtained from all subjects. Verbal consent was witnessed and formally recorded.

\section{References}

1. Marshall S, Bauer J \& Isenring E (2014) The consequences of malnutrition following discharge from rehabilitation to the community: a systematic review of current evidence in older adults. J Hum Nutr Diet 27, 133-141.

2. van Bokhorst-de van der Schueren MA, Guaitoli PR, Jansma EP et al. (2014) A systematic review of malnutrition screening tools for the nursing home setting. J Am Med Dir Assoc 15, 171-184.

3. van Bokhorst-de van der Schueren MA, Guaitoli PR, Jansma EP et al. (2014) Nutrition screening tools: does one size fit all? A systematic review of screening tools for the hospital setting. Clin Nutr 33, 39-58.

4. Correia M (2017) Nutrition screening vs nutrition assessment: what's the difference? Nutr Clin Pract. Published online: 14 December 2017. doi: 10.1177/ 0884533617719669.

5. Kvamme JM, Grønli O, Jacobsen BK et al. (2015) Risk of malnutrition and zinc deficiency in community-living elderly men and women: the Troms $\varnothing$ Study. Public Health Nutr 18, 1907-1913.

6. Westergren A, Hagell P \& Sjodahl Hammarlund C (2014) Malnutrition and risk of falling among elderly without home-help service - a cross sectional study. J Nutr Health Aging 18, 905-911.

7. Winter J, Flanagan D, McNaughton SA et al. (2013) Nutrition screening of older people in a community general practice, using the MNA-SF. J Nutr Health Aging 17, 322-325.

8. US Department of Health and Human Services, Centers for Disease Control and Prevention/National Center for Health Statistics (2016) Leading causes of death and numbers of deaths, by sex, race, and Hispanic origin: United States, 1980 and 2014 https://www.cdc.gov/nchs/data/hus/hus15. pdf (accessed December 2017).

9. Whitman S, Silva A, Shah A et al. (2004) Diversity and disparity: GIS and small-area analysis in six Chicago neighborhoods. J Med Syst 28, 397-411.

10. Gabrielson DK, Scaffidi D, Leung E et al. (2013) Use of an abridged scored Patient-Generated Subjective Global Assessment (abPG-SGA) as a nutritional screening tool for cancer patients in an outpatient setting. Nutr Cancer $\mathbf{6 5}$, 234-239.

11. Boulos C, Salameh P, Barberger-Gateau P (2016) Malnutrition and frailty in community dwelling older adults living in a rural setting. Clin Nutr 35, 138-143.

12. Detsky AS, McLaughlin JR, Baker JP et al. (1987) What is subjective global assessment of nutritional status? JPEN J Parenter Enteral Nutr 11, 8-13.

13. Sinnett S, Bengle R, Brown A et al. (2010) The validity of Nutrition Screening Initiative DETERMINE Checklist responses in older Georgians. J Nutr Elder 29, 393-409.

14. Wijnhoven HA, Schilp J, van Bokhorst-de van der Schueren MA et al. (2012) Development and validation of criteria for determining undernutrition in community-dwelling older men and women: the Short Nutritional Assessment Questionnaire 65+. Clin Nutr 31, 351-358.

15. Katz S (1983) Assessing self-maintenance: activities of daily living, mobility, and instrumental activities of daily living. $J$ Am Geriatr Soc 31, 721-727. 
16. US Census Bureau (2018) American Community Survey. https://www.census.gov/programs-surveys/acs/ (accessed February 2018).

17. Gunduz E, Eskin F, Gunduz M et al. (2015) Malnutrition in community-dwelling elderly in Turkey: a multicenter, crosssectional study. Med Sci Monit 21, 2750-2756.

18. Kiesswetter E, Pohlhausen S, Uhlig K et al. (2013) Malnutrition is related to functional impairment in older adults receiving home care. J Nutr Health Aging 17, 345-350.

19. Johansson L, Sidenvall B, Malmberg B et al. (2009) Who will become malnourished? A prospective study of factors associated with malnutrition in older persons living at home. $J$ Nutr Health Aging 13, 855-861.

20. Johansson Y, Bachrach-Lindstrom M, Carstensen $\mathrm{J}$ et al. (2009) Malnutrition in a home-living older population: prevalence, incidence and risk factors. A prospective study. J Clin Nurs 18, 1354-1364.

21. Covinsky KE, Covinsky MH, Palmer RM et al. (2002) Serum albumin concentration and clinical assessments of nutritional status in hospitalized older people: different sides of different coins? I Am Geriatr Soc 50, 631-637.

22. Rasheed S \& Woods RT (2013) Malnutrition and associated clinical outcomes in hospitalized patients aged 60 and older: an observational study in rural Wales. J Nutr Gerontol Geriatr 32, 71-80.

23. Sheean PM, Peterson SJ, Chen Y et al. (2013) Utilizing multiple methods to classify malnutrition among elderly patients admitted to the medical and surgical intensive care units (ICU). Clin Nutr 32, 752-757.

24. Mahan LK \& Raymond JL (2017) Krause's Food \& the Nutrition Care Process, 14th ed. St. Louis, MO: Elsevier.

25. US Census Bureau (2017) The nation's older population is still growing, Census Bureau reports. https://census.gov/ newsroom/press-releases/2017/cb17-100.html (accessed January 2018)

26. Institute of Medicine, Food and Nutrition Board (n.d.) Nutrition and Healthy Aging in the Community: Workshop Summary (2012). https://commed.vcu.edu/Chronic_Disease/ aging/healthaging_IOM.pdf (accessed October 2018).

27. Jensen GL, Cederholm T, Correia MITD et al. (2018) GLIM criteria for the diagnosis of malnutrition: a consensus report from the global clinical nutrition community. JPEN J Parenter Enteral Nutr. Published online: 2 September 2018. doi: 10.1002/jpen.1440.

28. Burge K \& Gazibarich B (1999) Nutritional risk among a sample of community-living elderly attending senior citizens' centres. Aust J Nutr Diet 56, 137-143.

29. Benedict J WD, Snow G \& Read M (1999) Use of nutrition screening to develop and target nutrition education interventions for Nevada's elderly. J Nutr Elder 19, 31-47.

30. Nykanen I, Lonnroos E, Kautiainen H et al. (2013) Nutritional screening in a population-based cohort of community-dwelling older people. Eur J Public Health 23, 405-409.

31. Lord JM, Midwinter MJ, Chen YF et al. (2014) The systemic immune response to trauma: an overview of pathophysiology and treatment. Lancet 384, 1455-1465.

32. Prado CM, Wells JC, Smith SR et al. (2012) Sarcopenic obesity: a critical appraisal of the current evidence. Clin Nutr 31, 583-601.

33. Batsis JA, Mackenzie TA, Jones JD et al. (2016) Sarcopenia, sarcopenic obesity and inflammation: results from the
1999-2004 National Health and Nutrition Examination Survey. Clin Nutr 35, 1472-1483.

34. Tappenden KA, Quatrara B, Parkhurst ML et al. (2013) Critical role of nutrition in improving quality of care: an interdisciplinary call to action to address adult hospital malnutrition. J Acad Nutr Diet 113, 1219-1237.

35. Hamilton C \& Boyce VJ (2013) Addressing malnutrition in hospitalized adults. JPEN J Parenter Enteral Nutr 37, 808-815.

36. Kellett J, Kyle G, Itsiopoulos C et al. (2016) Malnutrition: the importance of identification, documentation, and coding in the acute care setting. J Nutr Metab 2016, 9026098.

37. Cederholm T \& Jensen GL (2017) To create a consensus on malnutrition diagnostic criteria. JPEN J Parenter Enteral Nutr 41, 311-314.

38. Hamirudin AH, Charlton K \& Walton K (2016) Outcomes related to nutrition screening in community living older adults: a systematic literature review. Arch Gerontol Geriatr 62, 9-25.

39. Herndon AS (1995) Using the Nutrition Screening Initiative to survey the nutritional status of clients participating in a home delivered meals program. J Nutr Elder 14, 15-29.

40. Keller HH (2006) Meal programs improve nutritional risk: a longitudinal analysis of community-living seniors. J Am Diet Assoc 106, 1042-1048.

41. Keller HH, Haresign H \& Brockest B (2007) Process evaluation of bringing nutrition screening to seniors in Canada (BNSS). Can J Diet Pract Res 68, 86-91.

42. Southgate KM, Keller HH \& Reimer HD (2010) Determining knowledge and behaviour change after nutrition screening among older adults. Can J Diet Pract Res 71, 128-133.

43. Klein GL, Kita K, Fish J et al. (1997) Nutrition and health for older persons in rural America: a managed care model. $J \mathrm{Am}$ Diet Assoc 97, 885-888.

44. Leggo M, Banks M, Isenring $\mathrm{E}$ et al. (2008) A quality improvement nutrition screening and intervention program available to Home and Community Care eligible clients. Nutr Diet 65, 162-167.

45. Weddle DO, Wellman NS \& Bates GM (1997) Incorporating nutrition screening into three Older Americans Act elderly nutrition programs. J Nutr Elder 17, 19-37.

46. Wunderlich S, Bai Y \& Piemonte J (2011) Nutrition risk factors among home delivered and congregate meal participants: need for enhancement of nutrition education and counseling among home delivered meal participants. J Nutr Health Aging 15, 768-773.

47. Watson S, Zhang Z \& Wilkinson TJ (2010) Nutrition risk screening in community living older people attending medical or fall prevention services. Nutr Diet 67, 84-89.

48. Hamirudin AH, Walton K, Charlton K et al. (2017) Feasibility of home-based dietetic intervention to improve the nutritional status of older adults post-hospital discharge. Nutr Diet 74, 217-223.

49. Elia M, Parsons EL, Cawood AL et al. (2017) Costeffectiveness of oral nutritional supplements in older malnourished care home residents. Clin Nutr 37, 651-658.

50. van der Pols-Vijlbrief R, Wijnhoven HA, Schaap LA et al. (2014) Determinants of protein-energy malnutrition in community-dwelling older adults: a systematic review of observational studies. Ageing Res Rev 18, 112-131.

51. Peduzzi P, Concato J, Kemper E et al. (1996) A simulation study of the number of events per variable in logistic regression analysis. J Clin Epidemiol 49, 1373-1379. 